\title{
Web watch
}

A SOURCE OF TRUSTED TECHNIQUES

- http://www.cshprotocols.org/

If you are looking for information on methods, head for the Cold Spring Harbor (CSH) Protocols. CSH Laboratory is renowned for its teaching of biomedical research techniques. For decades, participants in the hands-on courses and users of its laboratory manuals have gained access to the most reliable methods in molecular and cellular biology. Now this access is moving online (Cold Spring Harbor Laboratory Press News Release, 8 March 2006).

CSH Protocols is created by $\mathrm{CSH}$ Laboratory Press in association with HighWire Press of Stanford University. This "new, interactive online methods database" will launch in June 2006 (Cold Spring Harbor Laboratory Press News Release, 8 March 2006). CSH Protocols promises to be an interactive source of new and classic research techniques, and will feature protocols in cell and molecular biology, genetics, protein science, imaging and bioinformatics.

The database will be fully searchable. The protocols themselves will be presented step-by-step and edited in the style that has made many other $\mathrm{CSH}$ manuals essential to the work of scientists worldwide. A moderated, interactive function will enable users to ask questions, discuss their own experiences and contribute suggestions. New protocols will be added monthly, and many leading laboratories and researchers have agreed to contribute their own methods. The launch collection of over 500 protocols is estimated to contain more than 900 protocols by the end of 2006. By signing up for the site's alerting services, registered users can keep abreast of the latest developments in their particular fields of interest.

CSH Protocols will be available through institutional site license and, prior to the official launch, librarians and scientists will have the opportunity to explore the utility and functions of the database. 